\title{
Anti-breast cancer drug screening based on Neural Networks and QSAR model
}

\begin{abstract}
Breast cancer is one of the most lethal cancers, estrogen receptor $\alpha$ Subtype $(\mathrm{ER} \alpha)$ is an important target. The compounds that able to fight ER $\alpha$ active may be candidates for treatment of breast cancer. The drug discovery process is a very large and complex process that often requires one selected from a large number of compounds. This paper considers the independence, coupling, and relevance of bioactivity descriptors, selects the 15 most potentially valuable bioactivity descriptors from 729 bioactivity descriptors. An optimized back propagation neural network is used for $\mathrm{ER} \alpha$, The pharmacokinetics and safety of 15 selected bioactivity descriptors were verified by gradient lifting algorithm. The results showed that these 15 biological activity descriptors could not only fit well with the nonlinear relationship of ER $\alpha$ activity can also accurately predict its pharmacokinetic characteristics and safety, with an average accuracy of $89.92 \sim 94.80 \%$. Therefore, these biological activity descriptors have great medical research value.
\end{abstract}

Volume 12 Issue $6-202$ I

\author{
Renxiong Xie,' Xia Jiang,' Bin Zhao' \\ 'School of Science, Hubei University of Technology, Wuhan, \\ Hubei, China \\ ${ }^{2}$ Hospital, Hubei University of Technology, Wuhan, Hubei, China
}

Correspondence: Dr. Bin Zhao, School of Science, Hubei University of Technology, Wuhan, Hubei, China,

Tel/Fax+86 I30 285I 7572, Email zhaobin835@nwsuaf.edu.cn

Received: December 0I, 202I | Published: December 20, 2021

Keywords: breast cancer, softmax function, adam algorithm, biological activity

\section{Introduction}

Breast cancer is one of the most common malignant tumors in women, and a malignant tumor occurring in ductal epithelium of the breast. It is the second leading cause of death by cancer among women in the United States. The total cost of illness for breast cancer has been estimated to be $\$ 3.8$ billion, of which $\$ 1.8$ billion represents medical care costs. ${ }^{1}$ The difficulty in drug development is one of the huge treatment costs. How to reasonably choose the appropriate cancer drugs from tens of thousands of compounds, is one of the effective ways to reduce the cost of breast cancer treatment. According to Anthony Howell study, Estrogen may stimulate the growth and progression of infiltrating tumours. ${ }^{2}$ Duan's study found that the estrogen is involved in the growth and differentiation of mammary epithelial cells in hormone dependent tumors. It plays an important role in the occurrence and development of breast cancer. ${ }^{3}$ Zhang's study showed that estrogen mainly acts through the estrogen receptor expressed in the nucleus, that is, by binding with estrogen receptor (ER) to form a complex. ${ }^{4}$ Through survival analysis, Chen found that the ER $\alpha$ positive rates of invasive carcinoma, invasive lobular carcinoma and ductal carcinoma in situ were $59.5 \%, 78.9 \%$ and $63.6 \%$, respectively. ${ }^{5}$ Accordingly, antihormone therapy is commonly used in breast cancer patients with ER $\alpha$ expression, which controls estrogen levels through regulating estrogen receptor activity. ER $\alpha$ mediates the E2 up regulation of PI3K/Akt signaling pathway and promotes cell proliferation. ${ }^{6}$ So, compounds that can antagonize ER $\alpha$ activity may be candidates for treatment of breast cancer. For example, tamoxifen and renoxifene are the ER $\alpha$ antagonists for clinical treatment of breast cancer. ${ }^{7}$ In order to screen potential active compounds, a potential compound model is usually established to collect compounds and bioactive data by targeting the specific estrogen receptor subtype targets associated with breast cancer. The quantitative structure-activity relationship (QSAR) model of compounds was constructed with the biological activity descriptor as the independent variable and the biological activity of compounds as the dependent variable. The model was used to predict the new compound molecules with good biological activity or guide the structural optimization of existing active compounds. A compound that wants to become a candidate drug, besides having good biological activity (here refers to anti breast cancer activity), also needs to have good pharmacokinetics and safety in human body. Pharmacokinetics is the study of the movement of a drug through the compartments of the body and the transformations (activation and metabolism) that affect it. ${ }^{8}$ It is called ADMET property, including absorption, distribution, metabolism, excretion and toxicity. When determining the biological activity of a compound, it is also necessary to consider its ADMET properties as a comprehensive consideration. In this paper, the coupling degree between bioactivity descriptor and $\mathrm{ER} \alpha$ activity is verified by BP neural network. After determining that the screened bioactivity descriptors can indeed affect ER $\alpha$ activity to a great extent, the ADMET property of bioactivity descriptors is further verified.

\section{Overview of bp neural network}

Artificial neural network is widely used in pattern recognition, function approximation and so on. BP neural network is a multilayer feedforward network simulating human brain. It has good adaptability and training ability, belongs to nonlinear dynamic system, and including two processes: forward propagation of information and back propagation of error. BP neural network consists of three parts: input layer, hidden layer and output layer. The input layer receives the input information, and then transmits the information to the hidden layer. The hidden layer analyzes and processes the data. Finally outputs acceptable information through the output layer. This information is continuously corrected through the reverse propagation of error, which can make full use of the coupling between data. BP neural network shows excellent accuracy in many fields. Therefore, this paper selects neural network as the main prediction method. Whether it is regression network or prediction network, the setting of the hidden layer and the number of hidden nodes of the network is very important. Too few hidden layers and hidden nodes will lead to less data information that the neural network can process, resulting in low prediction accuracy, and too many hidden layers will lead to overfitting of the model. There is no general calculation formula for the setting of the optimal number of hidden nodes. It is more based on the empirical formula or changing the number of hidden nodes to continuously train the model to find the number of hidden nodes with the smallest error. ${ }^{9-11}$ Basic structure diagram of BP neural network is shown in Figure 1. 


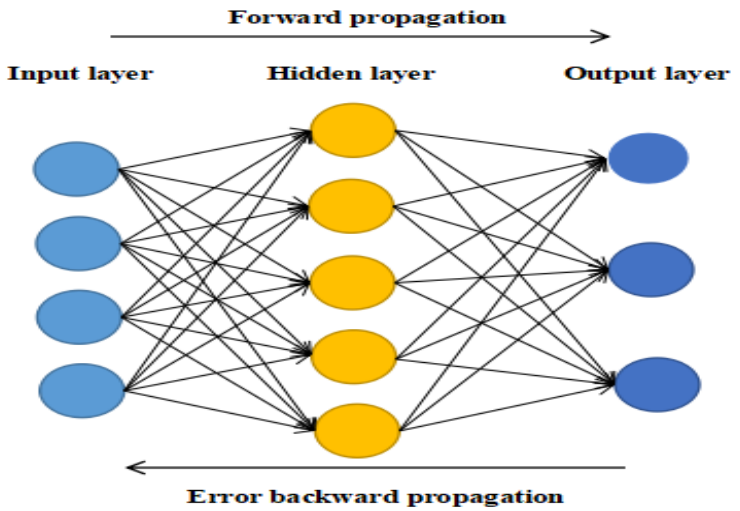

Figure I Basic structure diagram of BP neural network.

The activation function of BP neural network usually uses softmax function to give corresponding weight to each node and transfer information between nodes in the network. In addition, there is an offset weight in the propagation of each layer of network, which is an additional constant of softmax function. We will use softmax function as an activation function of the BP neural network. The softmax function as follow:

$$
S_{i}=\frac{e^{i}}{\sum_{j} e^{j}}
$$

Its operating principle is shown in Figure 2.

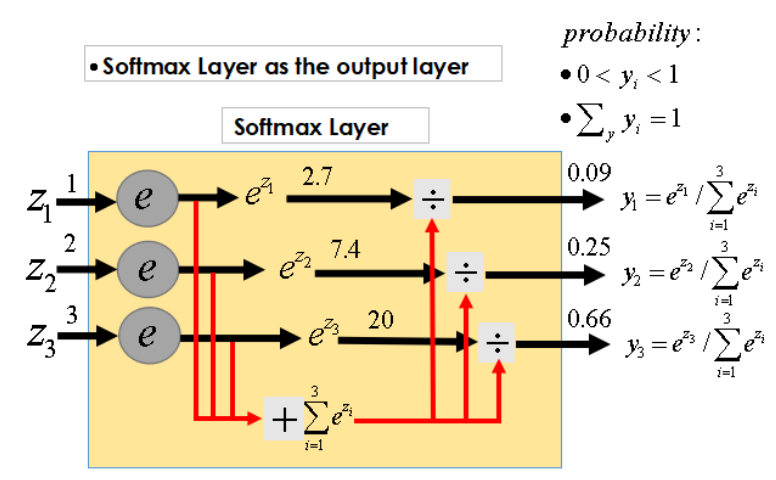

Figure 2 Principle of Softmax function.

The softmax function in the figure maps the input values between 0,1 with the sum of 1 , understanding these values as probabilities of probability, and the node with the highest probability can be selected as the prediction target. The BP neural network algorithm is as follows:

Enter layer to the hidden layer:

$$
\alpha_{h}=\sum_{i=1}^{d} v_{i h} * x_{i}
$$

The softmax activation function passing through the hidden layer:

$$
b_{h}=f\left(\alpha_{h}-\gamma_{h}\right)
$$

Hideen layer to the output layer:

$$
\beta_{j}=\sum_{h=1}^{q} w h_{j} * b_{h}
$$

Activation function passing through the output layer:

$$
y_{j}^{k^{\prime}}=f\left(\beta_{j}-\eta_{j}\right)
$$

Computing error:

$$
E_{k}=1 / 2 * \sum_{j=1}^{l}\left(y_{j}^{k^{\prime}}-y_{j}^{k}\right)^{2}
$$

Where $x_{i}$ is the input, $v_{i h}$ is the weight of the input layer to the hidden layer, $\gamma_{h}$ is the hidden layer valve value, $w_{h j}$ is the weights between hidden layer and output layer, $\eta_{j}$ is the input layer valve value, $f(\bullet)$ is the softmax activation function.

In the model training, the gradient optimization algorithm (Adam algorithm) is used to optimize the model to obtain the best results. ${ }^{12}$

Adam algorithm:

Initialize 1 st, 2 st moment vector and time step:

$$
m_{0} \cdot v_{0} . t \leftarrow 0
$$

do while:

$$
t \leftarrow t+1
$$

Computing the gradient:

$$
\mathrm{g}_{t} \leftarrow \nabla_{\theta} f\left(\theta_{t-1}\right)
$$

Update biased first moment estimate:

$$
m_{t} \leftarrow \beta_{1} * m_{t-1}+\left(1-\beta_{1}\right) * g_{t}
$$

Update biased second moment estimate:

$$
v_{t} \leftarrow \beta_{2} * v_{t-1}+\left(1-\beta_{2}\right) * g_{t}^{2}
$$

Compute bias-corrected first moment estimate:

$$
\hat{m}_{t} \leftarrow \frac{m_{t}}{\left(1-\left(\beta_{1}\right)^{t}\right)}
$$

Compute bias-corrected second moment estimate:

$$
\hat{v}_{t} \leftarrow \frac{v_{t}}{\left(1-\left(\beta_{2}\right)^{t}\right)}
$$

Update parameters:

$$
\theta_{t} \leftarrow \theta_{t-1}-\alpha * \frac{\hat{m}_{t}}{\sqrt{\hat{v}_{t}}+\varepsilon}
$$

Where $\alpha$ is the step length, $\beta_{1} ; \beta_{2}$ is the momen estimation of exponential decay rate, and $f(\theta)$ is the random objective function of parameter $\theta$. Adam algorithm will be used to optimize the parameters of BP neural network in order to accelerate convergence and improve accuracy. The model is:

Step 1: Initialize the network weight and bias, give each network connection weight a small random number, and each neuron with a bias will also be initialized to a random number.

Step 2: Forward propagation. Input a training sample, and then calculate the output of each neuron. The calculation method of each neuron is the same, which is obtained by the linear combination of its inputs. 
Step 3: The gradient descent method is used to calculate the error and carry out back propagation. The weight gradient of each layer is equal to the input of the connection of the previous layer multiplied by the weight of the layer and the reverse output of the connection of the next layer.

Step 4: The weight gradient in the third step is used to adjust the network weight and neural network bias.

Step 5: Back propagation, Adam algorithm is used to accelerate the weight adjustment, initialize the moment vector and exponential weighted infinite norm to 0 , update the parameters through vector operation, and iterate in t time from step size to 1 . Sort errors and return.

Step 6: At the end of judgment, for each sample, judge if the error is less than the threshold set by us or has reached the number of iterations. We'll finish training, otherwise, return step 2.

\section{Data description and preprocessing}

In this paper, the bioactivity description data set is used to verify the ER $\alpha$ activity and ADMET properties respectively. The description dataset contains 729 biological activity descriptors of 1974 compounds. Because the data dimension is too large and contains a large number of repetitions and useless variables, this paper selects 15 most representative biological activity descriptors from the 729 biological activity descriptors of 1974 compounds. Firstly, low variance filtering is used to delete the biological activity descriptors with low information, then considering the correlation and independence between variables, Lasso regression is used to select these variables, and finally considering the coupling degree between variables and $\mathrm{ER} \alpha$ activity. The final 15 most representative biological activity descriptors are obtained. The specific steps are as follows:

Step 1: Because the variance of variable can reflect the degree of dispersion, the variable with small variance contains little information, which can not provide key and useful information for the construction of the model. Therefore, for 729 biological activity descriptors of 1974 compounds, the variance of 729 variables is calculated and arranged from large to small.

Step 2: After cleaning the biological activity descriptors with low information or no information, use the remaining molecular descriptors to further process the repeated information of the data, so as to make the data relatively independent. In this paper, Lasso ${ }^{13}$ feature selection method is used to propose a variable from two variables with strong correlation to eliminate duplicate information. The essence of lasso feature selection method is to seek the sparse expression of the model and compress the coefficients of some features to 0 , so as to achieve the purpose of feature selection. The parameter estimation of lasso feature selection method is as follows:

$$
\hat{\gamma}=\underset{\gamma}{\arg \min ^{2}}\left\|y-\sum_{i=1}^{p} x_{i} \gamma_{i}\right\|^{2}+\lambda \sum_{i=1}^{p}\left|\gamma_{i}\right|
$$

$\lambda$ is a nonnegative regular parameter, which represents the complexity of the model. The greater its value, the greater the penalty of the linear model, the size of the regularization parameters was determined by cross-validation.

Step 3: Spearman rank correlation coefficient is a nonparametric index to measure the dependence of two variables, which can reflect the coupling degree between variables. This paper uses Spearman rank correlation coefficient to obtain the final 15 representative biological activity descriptors
Three screening processes by Figure 3 shows.

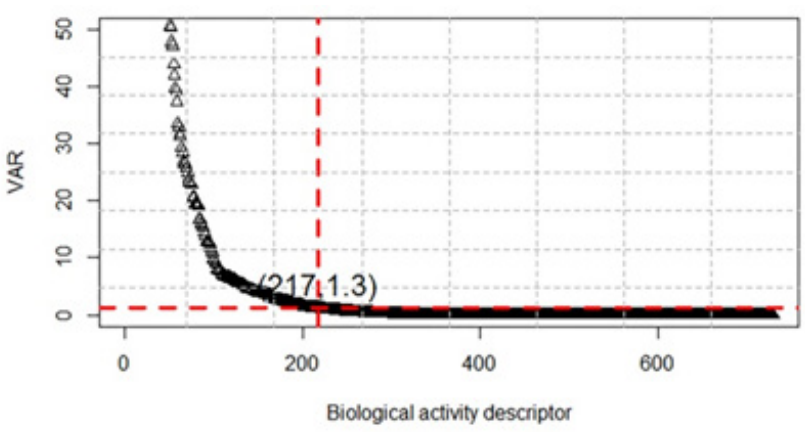

(a) Step 1 .

$\begin{array}{llllllllllllllll}191 & 178 & 153 & 137 & 123 & 101 & 80 & 60 & 52 & 44 & 34 & 21 & 11 & 7 & 3 & 0\end{array}$

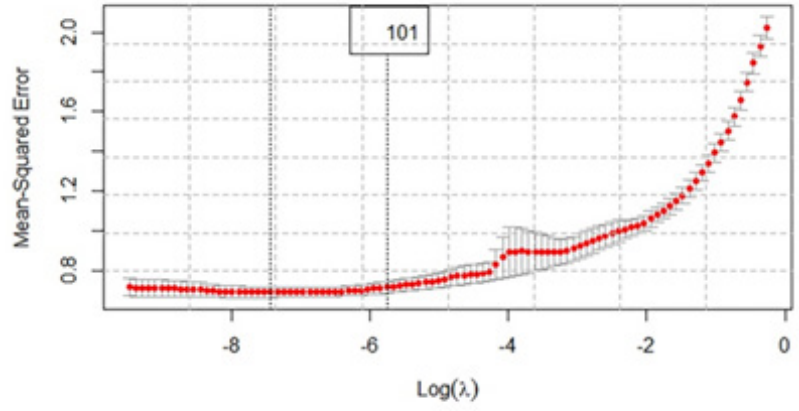

(b) Step 2 .

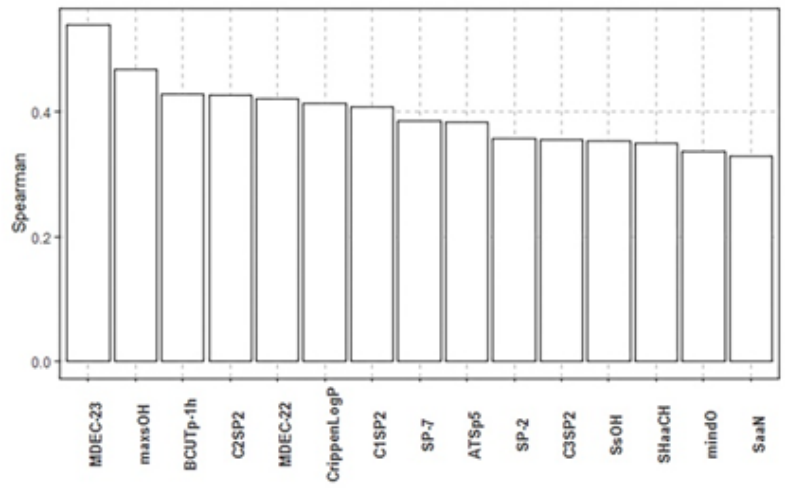

(e) Step 3

Figure 3 Three screening processes.

In step 1, 217 biological activity descriptors with variance greater than 1.3 were left.

In step 2, 101 bioactivity descriptors were retained by lasso feature selection.

In step 3, 101 biological activity descriptors are sorted according to Spearman rank correlation coefficient, leaving the most representative 15 biological activity descriptors. The final screening results are shown in Table 1.

ADMET properties are composed of five aspects: absorption, distribution, metabolism, excretion and toxicity.

The corresponding values are provided in the form of two classifications, ' 1 ' represents good or yes, and ' 0 ' represents poor or no. Comparison table of ADMET properties are shown in Table 2. 
Table I Comparison table of Biological activity descriptor

\begin{tabular}{|c|c|}
\hline Variable & Variable interpretation \\
\hline MDEC-23 & $\begin{array}{l}\text { Molecular distance edge between all secondary and } \\
\text { tertiary nitrogens. }\end{array}$ \\
\hline $\operatorname{maxs} \mathrm{OH}$ & Maximum atom-type E-State: -OH. \\
\hline BCUTplh & nlow highest polarizability weighted BCUTS. \\
\hline $\mathrm{C} 2 \mathrm{SP} 2$ & Doubly bound carbon bound to two other carbons. \\
\hline MDEC22 & $\begin{array}{l}\text { Molecular distance edge between all secondary } \\
\text { carbons. }\end{array}$ \\
\hline Crippen LogP & Crippen's LogP. \\
\hline CISP2 & Doubly hound carbon bound to one other carbon. \\
\hline SP7 & Simple path, order 7. \\
\hline ATSp5 & $\begin{array}{l}\text { ATS autocorrelation descriptor, weighted by } \\
\text { polarizability. }\end{array}$ \\
\hline SP2 & Simple path, order 2. \\
\hline C3SP2 & Doubly bound carbon bound to three other carbons. \\
\hline $\mathrm{SsOH}$ & Sum of atom-type E-State: $-\mathrm{OH}$. \\
\hline $\mathrm{SHaaCH}$ & Sum of atom-type H E-State: $\mathrm{CH}$. \\
\hline $\operatorname{mindO}$ & Minimum atom-type E-State: $=0$. \\
\hline SaaN & Sum of atom-type E-State: $\mathrm{N}$. \\
\hline
\end{tabular}

Table 2 Comparison table of ADMET properties

\begin{tabular}{ll}
\hline Variable & Describe \\
\hline Caco-2 & Permeability of small intestinal epithelial cells. \\
CYP3A4 & Metabolize. \\
hERG & Cardiotoxic. \\
HOB & Oral bioavailability. \\
MN & Genotoxic. \\
\hline
\end{tabular}

\section{Model training and prediction}

In order to avoid over fitting and improve the generalization ability of the model, ${ }^{14}$ we cut the remaining 15 bioactivity descriptors into $80 \%$ of the training set and $20 \%$ of the test set. Considering the coupling and the nonlinear relationship between the data, the neural network is used for training and prediction, the training set is used to set the model parameters, and the test set is used to calculate the default accuracy and verify the rationality of the model. When training the model, we should also consider the convergence speed of the model. Neural network is a complex structure with large amount of calculation. When there are too many input variables in the input layer and the amount of data is too large, gradient optimization algorithm is usually used to accelerate the convergence speed of neural network. Adam algorithm is used for model optimization in this paper.The results are as follows:

As can be seen from Figure 4, The red line is the logarithm of $\mathrm{ER} \alpha$, the blue line is the regression prediction result of neural network with one hidden layer, and the black line is the regression prediction result of neural network with two hidden layers. Among them, when the hidden layer is 1 , the mean square error of prediction is 0.696 , and when the hidden layer is 2 , the mean square error of prediction is 0.759 . Obviously, when the hidden layer is 1 , the regression prediction result is more accurate, and the good prediction accuracy shows that the ER $\alpha$ activity can be controlled by controlling the 15 biological activity descriptors selected in this paper, so that we can inhibit the $\mathrm{ER} \alpha$ activity.

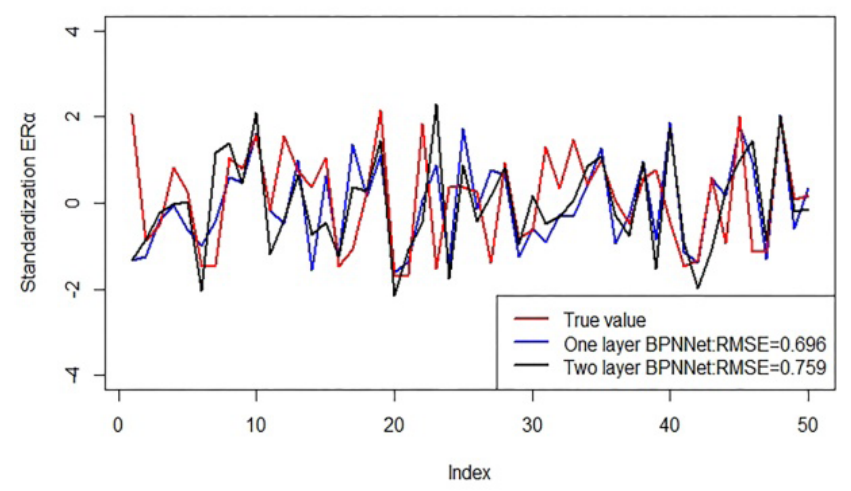

Figure 4 The predict of Adam-BPNNet.

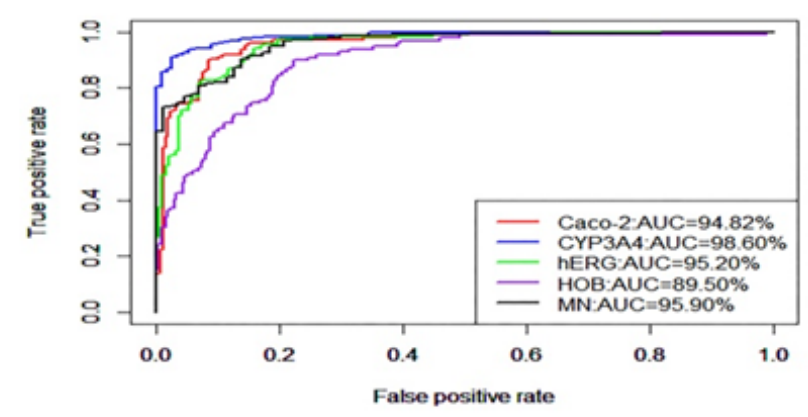

(a) XGBoost-ROC.

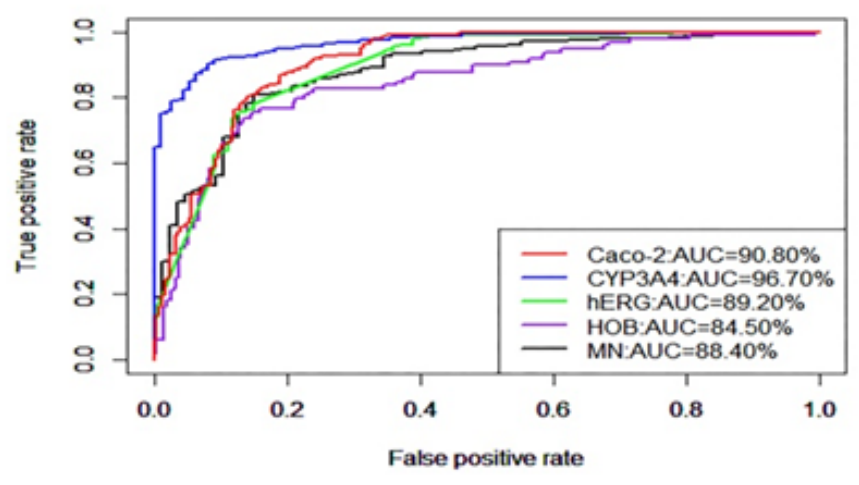

(b) One layer BPNNet-ROC.

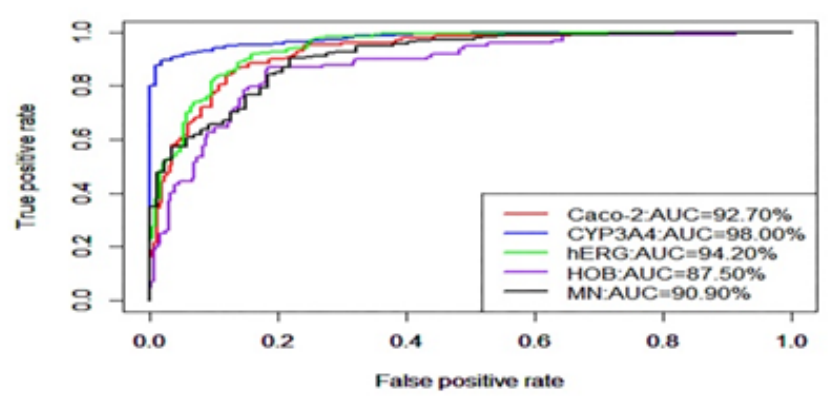

(c) GBM-ROC.

Figure 5 ROC curve of each classification method. 
In order to ensure that the selected bioactivity descriptors have good medical properties, the ADMET properties of these 15 bioactivity descriptors were verified. The commonly used machine learning methods are used for multiple prediction to eliminate contingency. ${ }^{15-17}$ ROC curve shown in Figure 4

It can be seen from Table 3 that the three models show very high prediction accuracy, as the table shows that the average accuracy of XGBoost is 0.948 , the average accuracy of GBM is 0.927 , the average accuracy of Adam-BPNNet is 0.899 . Among the five metric variables that determine the pharmacokinetics, as well as the safety properties. The three models show that CYP3A4 is highly coupled with 15 biological activity descriptors, the coupling degrees are 0.986 , 0.980 and 0.967 respectively. HOB is the lowest coupled with one biological activity descriptor, the coupling degrees are $0.895,0.875$ and 0.845 respectively. The pharmacokinetic and safety fit levels of the 15 biological activity descriptors selected in the article in the three models are between 0.8992 and 0.948 , which are relatively stable. The average expression of drug permeability, absorption, metabolism, cardiotoxicity, genotoxicity and 15 biological activity descriptors reached more than 0.872 . This shows that the 15 biological activity descriptors selected in this paper can not only reflect ER $\alpha$ activity to a great extent, it can also reflect good ADMET properties.

Table 3 Comparison table of AUC values of different classification models

\begin{tabular}{ll}
\hline Classifier & AUC \\
\hline XGBoost & Caco-2: 0.948 \\
& CYP3A4:0.986
\end{tabular}

hERG: 0.952

HOB: 0.895

$\mathrm{MN}: 0.959$

GBM

Caco-2: 0.927

CYP3A4: 0.980

hERG: 0.942

HOB: 0.875

$\mathrm{MN}: 0.909$

Adam-BPNNet

Caco-2: 0.908

CYP3A4: 0.967

hERG: 0.892

HOB: 0.845

$\mathrm{MN}: 0.884$

\section{Conclusion}

The results show that the 15 biological activity descriptors selected in this paper can predict ER $\alpha$ activity with a low mean square error of 0.676 , which indicates that there is a high coupling between them. In addition, they can also reflect the properties of ADMET at an average level of 0.8992 0.948, so they have good medical value. The development of anti breast cancer drugs is a complex and long process. In this process, it is necessary to test the effects of drugs containing various biological components on target cells. If all the combined drugs are tested, it will be a long process. In order to improve the development cycle and cost of anti breast cancer drugs, we can consider using these bioactive descriptors to synthesize breast cancer resistant compounds.

Because the experimental data are limited, the influence of these 15 bioactive descriptors on the activity of other target cells is not considered. Therefore, the bioactive descriptors selected in this paper have limitations in the effect of breast cancer. Furthermore, lasso feature selection method is used to screen bioactivity descriptors, which may omit some important bioactivity descriptors.

When the synthetic breast cancer drugs are synthesized, the best value or range of bioactive descriptors can further reduce the development cost and development cycle of anti breast cancer drugs. Therefore, in this paper, we can further study the best values of various bioactive descriptors. At the same time, we also hope that the variable screening method and validation method can be applied to more biopharmaceutical processes.

\section{Acknowledgments}

This work was supported by the Philosophical and Social Sciences Research Project of Hubei Education Department (19Y049), and the Staring Research Foundation for the Ph.D. of Hubei University of Technology (BSQD2019054), Hubei Province, China.

\section{Funding}

None.

\section{Conflicts of interest}

We have no conflict of interests to disclose and the manuscript has been read and approved by all named authors.

\section{References}

1. Elixhauser A. Costs of breast cancer and the cost-effectiveness of breast cancer screening. International Journal of Technology Assessment in Health Care. 1991;7(4):604-615.

2. Howell A. Clinical evidence for the involvement of oestrogen in the development and progression of breast cancer. Proceedings of the Royal Society of Edinburgh. Section B. Biological Sciences. 1989;95:49-57.

3. Duan XN, Bai WP. China consensus on the safety management of endometrial endometrium in patients with breast cancer treated with selective estrogen receptor modulators. Journal of Capital Medical University. 2021;42(4):672-677.

4. Zhang R, Du QL. Osteoglycin inhibits proliferation of Luminal breast cancer cells by up regulating estrogen receptor expression. Chinese Journal of basic and clinical medicine. 2021;28(1):1-7.

5. Chen Q, Meng G, Huang W. The prognostic relationship between ER and PR expression in breast cancer and affecting the positive rate in association. Journal of Clinical and Experimental Pathology. 2016;32(01):13-18.

6. Zhu XX, Yang HY. Estrogen receptor subtypes $\alpha$ and $\beta$ Expression and clinical significance in breast cancer. China Clinical Oncology and rehabilitation. 2014;21(6):766-768.

7. Liu R, Zhao J. Estrogen receptor subtype ER $\alpha$; ER $\beta$ Expression in breast cancer. China Journal of Gerontology. 2012;32(16):3389-3390.

8. Smith, T. Pharmacokinetics. Cambridge: Cambridge University Press; 2016:562-577.

9. Wang XM, Chen R, Qiao B. Application of BP neural network in tea disease classification and recognition. Guizhou Science. 2020;38(4):9396 
10. Cao YF, Zhao YJ. Research on computer intelligent image recognition echnology based on GA-BP Neural Network. Applied Laser. 2017;37(1):139-143.

11. Qi Y. Experimental Study on NDVI Inversion Using GPS-R Remote Sensing Based on BP Neural Network. Xuzhou: China University of Mining and Technology; 2018.

12. Kingma D, Ba J. A method for stochastic optimization. 3rd International Conference for Learning Representations. San Diego; 2015.

13. Li H. Statistical learning methods. Beijing: Tsinghua University Press; 2012.
14. Adcock B, Hansen A. The LASSO and its cousins. Cambridge University Press; 2021:129-141.

15. Ma BS, Ge Y, Chai BJ, et al. An improved survival prediction model based on Xgboost. Bioinformatics (Oxford, England). 2021;3(12):22-45.

16. Lombardi G, Bergo E, Bellu L. P01.021 Comprehensive geriatric assessment (CGA) for outcome prediction in elderly patients (PTS)with glioblastoma (GBM): a mono-institutional experience. Neuro-Oncology. 2018;20(suppl_3).

17. Korvink M, Martin J, Long M. Real-time identification of patients included in the CMS bundled payment care improvement (BPCI) program. Infection Control \& Hospital Epidemiology. 2020;41(S1):S367-S368. 